\title{
Tránsito de universidad docente a universidad de investigación. ¿Un problema de información académica, de taxonomías o de rankings universitarios?
} The transit of a teaching university to a research
university. A problem of academic information,
taxonomies or university rankings?

\author{
Atilio Bustos-González
}

Cómo citar este artículo:

Bustos-González, Atilio (2019). “Tránsito de universidad docente a universidad de investigación. ¿ Un problema de información académica, de taxonomías o de rankings universitarios?". El profesional de la información, v. 28, n. 4, e280422.

https://doi.org/10.3145/epi.2019.jul.22

Artículo recibido el 2 de septiembre de 2019

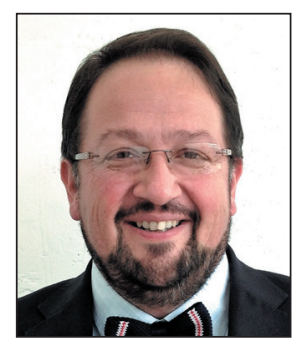

Atilio Bustos-González $ه$

https://orcid.org/0000-0002-0822-2869

SCImago Research Group

Bogotá, Colombia

atilio.bustos@scimago.es

\section{Resumen}

El objetivo de este artículo es generar una discusión en torno a cómo diferenciar universidades de investigación, universidades docentes que hacen investigación y universidades docentes. Se analiza la distinción que hacen las taxonomías universitarias, los rankings globales de educación superior y la información académica generada por los propios actores del sistema. El estudio demuestra como actualmente las taxonomías y modelos tradicionales no caracterizan los sistemas universitarios, ni siquiera en el país donde nacieron. Los rankings globales de universidades muestran ser una fuente útil de información y una forma de contribuir a la visibilidad y reputación institucional. La información académica, cuando se trata de indicadores cualitativos de la producción científica, encuentra limitaciones de acceso, especialmente para universidades docentes que hacen investigación. Se documentan ejemplos de falta de rigor metodológico cuando se reutilizan dichos indicadores. La caracterización de la producción científica debe equilibrar los indicadores que describen el tamaño de la producción, con aquellos que caracterizan la performance, impacto y excelencia. El análisis de la información empírica muestra que algunas instituciones, de todos los países analizados y de todos los tamaños, presentan resultados de impacto que están descendidos de la media del mundo, evidenciando que no existe una correlación positiva entre desempeño investigador y tamaño institucional. La dificultad no radica tanto en determinar cuándo estamos frente a una universidad de investigación, sino en definir la frontera entre una universidad docente que hace investigación y la que es esencialmente docente. Como mínimo una universidad docente que hace investigación produce 100 artículos por año, contados en ventanas de cinco años, siendo deseable que ese umbral de tamaño esté por encima de los 150 artículos anuales. Una universidad de investigación produce como mínimo entre 1.000 y 2.000 artículos por año y gradúa al menos 20 doctores por año. En ambos casos, las instituciones deben alcanzar unos indicadores de performance, impacto y excelencia, ambos liderados, que demuestren que la universidad cuenta con un claustro de profesores en condiciones de hacer investigación original de forma autónoma, con un nivel de resultados equivalentes a los de sus colegas en el mundo.

\section{Palabras clave}

Universidad de investigación; Universidad docente que hace investigación; Universidad docente; Taxonomías universitarias; Rankings globales de universidades; Rankings de universidades; Información académica. 


\begin{abstract}
The objective of this article is to generate a discussion about how to differentiate research universities, teaching universities that do research and teaching universities. The distinction made by university taxonomies, global higher education rankings and the academic information generated by the system's own actors is analyzed. The study demonstrates how currently taxonomies and traditional models do not characterize university systems, even in the country where they were born. The global rankings of universities prove to be a useful source of information and a way to contribute to institutional visibility and reputation. Academic information, when it comes to qualitative indicators of scientific production, finds access limitations, especially for teaching universities that carry out research. Examples of lack of methodological rigor are documented when these indicators are reused. The characterization of scientific production must balance the indicators that describe the size of the production, with those that characterize performance, impact and excellence. The analysis of the empirical information shows that some institutions, of all countries analyzed and of all sizes, have impact results that are below the world average, showing that there is no positive correlation between research performance and institutional size. The difficulty is not so much in determining when we are in front of a research university, but in defining the border between a teaching university that does research and the one that is essentially teaching. $A$ teaching university that does research produces at least 100 articles per year, counted in five-year windows, being desirable that this size threshold be above 150 annual articles. A research university produces at least 1,000 to 2,000 articles per year and graduates at least 20 doctors per year. In both cases, the institutions must reach performance, impact and excellence indicators, both led, that demonstrate that the university has a faculty staff able to do original research autonomously, with a level of results equivalent to those of their colleagues in the world.
\end{abstract}

\title{
Keywords
}

Research university; Teaching university that does research; Teaching university; University taxonomies; Global rankings of universities; University rankings; Academic information.

\section{Introducción}

¿Cuándo una institución de educación superior pasa de ser una universidad docente y es percibida como una universidad de investigación? Sin duda, ésta es una pregunta crítica para rectores, comunidades universitarias, organismos internacionales y nacionales de acreditación, ministerios de ciencia y de educación, e instituciones generadoras de rankings universitarios, entre otros actores relevantes.

El análisis de las afiliaciones de los autores que aparecen en revistas académicas revela que la mayoría de los documentos publicados durante el período 2014-2018 pueden atribuirse a trabajos de investigación realizados en universidades, una observación ya advertida en los trabajos de De-Moya-Anegón et al. (2014), Daraio et al. (2015), González-Albo et al. (2016), y Patra y Muchie (2018). En los países de Europa Occidental el sector universidades participa ${ }^{1}$ en un 73,6\% de la producción científica, en América del Norte incluido México el sector constituye el 79,8\% y en América del Sur el 86,5\% (en la tabla 1 se muestran los valores desagregados por países). Evidentemente se están comparando dominios científicos de diferentes tamaños y características, lo cual se denota al comparar los esfuerzos relativos desplegados por la producción de investigadores situados en los sectores salud, público, privado y otros² (tabla 1) (De-Moya-Anegón et al., 2014; OCDE, 2016).

Tabla 1. Proporción de producción científica participada por sectores en 2014-2018

\begin{tabular}{|c|c|c|c|c|c|c|}
\hline País & $\begin{array}{c}\text { Producción total } \\
\text { 2014-2018 }\end{array}$ & $\begin{array}{l}\text { \% Producción } \\
\text { participada sector } \\
\text { Educación supe- } \\
\text { rior 2014-2018 }\end{array}$ & $\begin{array}{l}\text { \% Producción } \\
\text { participada } \\
\text { sector Salud } \\
2014-2018\end{array}$ & $\begin{array}{l}\text { \% Producción } \\
\text { participada } \\
\text { sector Público } \\
\text { 2014-2018 }\end{array}$ & $\begin{array}{c}\text { \% Producción } \\
\text { participada sector } \\
\text { Privado } \\
\text { 2014-2018 }\end{array}$ & $\begin{array}{c}\text { \% Producción } \\
\text { participada sector } \\
\text { Otros } \\
\text { 2014-2018 }\end{array}$ \\
\hline Brasil & 375.040 & 90,78 & 8,15 & 10,49 & 1,08 & 0,48 \\
\hline Chile & 64.804 & 90,63 & 6,53 & 6,56 & 0,41 & 6,22 \\
\hline Colombia & 52.621 & 89,16 & 10,05 & 2,94 & 0,52 & 2,60 \\
\hline Canadá & 539.239 & 82,58 & 17,23 & 5,04 & 0,42 & 0,78 \\
\hline Reino Unido & 1.029 .289 & 80,36 & 14,70 & 4,02 & 1,84 & 0,30 \\
\hline Holanda & 303.353 & 79,56 & 34,39 & 10,02 & 1,42 & 0,24 \\
\hline Estados Unidos & 3.379 .682 & 78,77 & 24,09 & 8,03 & 3,79 & 1,48 \\
\hline México & 116.058 & 77,91 & 15,86 & 19,98 & 1,08 & 0,72 \\
\hline Italia & 573.874 & 77,31 & 14,13 & 17,73 & 0,90 & 1,81 \\
\hline Argentina & 70.394 & 75,27 & 8,94 & 64,14 & 0,41 & 0,81 \\
\hline España & 467.205 & 73,21 & 24,03 & 22,30 & 1,45 & 1,73 \\
\hline Alemania & 894.042 & 65,66 & 13,42 & 25,87 & 2,95 & 0,96 \\
\hline Francia & 613.664 & 65,27 & 21,04 & 52,28 & 2,47 & 0,99 \\
\hline
\end{tabular}

Fuente de datos: Scopus. Análisis: SCImago Lab 
En todos los países comparados, independientemente de lo diversos que pueden ser en el diseño de sus sistemas científicos, el sector Educación superior participa en más del $65 \%$ de los resultados de investigación. Argentina y Francia, donde el Conacyt y el Cnrs, respectivamente, muestran una actividad muy dinámica, la mayor parte de los investigadores activos se localizan en las universidades y muestran en sus resultados de investigación una multiafiliación: a la universidad donde investiga y realiza docencia, y al organismo nacional de ciencia y tecnología (tabla 1).

Tabla 2. Número de instituciones de educación superior por rango de producción científica en 2018

\begin{tabular}{|c|c|c|c|c|c|c|c|c|c|c|c|}
\hline País & $\begin{array}{l}\text { \# de instituciones } \\
\text { educ. superior } \\
\text { con producción en } \\
2018\end{array}$ & $\begin{array}{c}25.000 \mathrm{a} \\
18.001 \\
\text { docs. }\end{array}$ & $\begin{array}{l}18.000 \\
\text { a } \quad 12.001 \\
\text { docs. }\end{array}$ & $\begin{array}{c}12.000 \\
\text { a } 6.001 \\
\text { docs. }\end{array}$ & $\begin{array}{c}6.000 \text { a } \\
4.001 \\
\text { docs. }\end{array}$ & $\begin{array}{c}4.000 \text { a } \\
1.001 \\
\text { docs. }\end{array}$ & $\begin{array}{l}1.000 \\
\text { a } 501 \\
\text { docs. }\end{array}$ & $\begin{array}{c}500 \text { a } \\
101 \\
\text { docs. }\end{array}$ & $\begin{array}{l}100 \\
\text { a } 21 \\
\text { docs. }\end{array}$ & $\begin{array}{l}20 \text { a } 1 \\
\text { docs. }\end{array}$ & $\begin{array}{l}\% \text { instituciones } \\
\text { con } 100 \text { o menos } \\
\text { documentos }\end{array}$ \\
\hline México & 224 & & & 1 & 0 & 5 & 6 & 31 & 34 & 147 & 80,8 \\
\hline Colombia & 153 & & & & & 3 & 2 & 31 & 49 & 68 & 76,5 \\
\hline Argentina & 94 & & & & & 3 & 4 & 18 & 29 & 40 & 73,4 \\
\hline Brasil & 401 & & 1 & 0 & 4 & 25 & 17 & 80 & 108 & 166 & 68,3 \\
\hline Holanda & 35 & & & 4 & 3 & 6 & 0 & 6 & 7 & 9 & 45,7 \\
\hline $\begin{array}{l}\text { Estados } \\
\text { Unidos }\end{array}$ & 786 & 1 & 5 & 32 & 22 & 110 & 68 & 222 & 218 & 108 & 41,5 \\
\hline Chile & 58 & & & & & 3 & 7 & 24 & 9 & 15 & 41,4 \\
\hline España & 94 & & & & 3 & 30 & 13 & 19 & 14 & 15 & 30,9 \\
\hline Alemania & 118 & & & 1 & 6 & 40 & 14 & 23 & 22 & 12 & 28,8 \\
\hline Francia & 157 & & & 1 & 6 & 21 & 33 & 57 & 20 & 19 & 24,8 \\
\hline Canadá & 66 & & 2 & 2 & 5 & 17 & 9 & 19 & 6 & 6 & 18,2 \\
\hline $\begin{array}{l}\text { Reino } \\
\text { Unido }\end{array}$ & 133 & & 2 & 5 & 12 & 36 & 27 & 35 & 12 & 4 & 12,0 \\
\hline Italia & 76 & & & 3 & 6 & 29 & 19 & 12 & 2 & 5 & 9,2 \\
\hline
\end{tabular}

Umbral: sólo se incluyeron instituciones que registraron al menos 1 documento en 2018.

Fuente de datos: Scopus. Análisis: SCImago Lab

La diversidad de las capacidades de producción científica de las instituciones del sector Educación superior de los países desplegados muestra una alta dispersión: México (80,8\%), Colombia (76,5\%), Argentina (73,4\%) y Brasil $(68,3 \%)$, tienen una alta proporción de instituciones que no superan los 100 artículos científicos por año (tabla 2).

Si la mayor parte de la investigación científica se genera en las universidades, y las universidades presentan niveles de desarrollo muy diferentes entre sí, para diferentes actores tiene importancia clasificarlas de acuerdo a su capacidad de generar ciencia.

\section{Taxonomías universitarias}

Las taxonomías universitarias son intentos de clasificar la diversidad de universidades que integran un sistema de educación superior dado, de forma que se facilite a diferentes actores su comprensión y toma de decisiones. Al basarse en un conjunto reducido de atributos, las taxonomías tienen el riesgo de no reconocer adecuadamente la diversidad, cultura organizacional, tamaños, extensión geográfica e historia de las instituciones sobre las cuales se aplica. A pesar de estas limitaciones, las taxonomías de universidades son ampliamente utilizadas, discutidas, y con cierta frecuencia se proponen nuevas taxonomías. Algunas de estas propuestas son interaccionadas (buscan favorecer una o unas instituciones sobre otras) y otras sólo tienen validez nacional, especialmente porque los datos utilizados para construirlas sólo están disponibles en un dominio determinado. En este sentido las taxonomías experimentan las mismas limitaciones de los rankings universitarios (Brunner, 2009).

Brunner (2007) propone un conjunto de factores que han sido tenidos en cuenta por varios autores para la elaboración de taxonomías de instituciones universitarias, en las que incluye:
Si la mayor parte de la investigación científica se genera en las universidades, y las universidades presentan niveles de desarrollo muy diferentes entre sí, tiene importancia poder clasificarlas de acuerdo a su capacidad de generar ciencia 
- control, gestión y financiamiento;

- cobertura de áreas de conocimiento;

- funciones;

- tamaño;

- localización y alcance;

- modalidad de provisión docente;

- reputación o prestigio.

El factor funciones es donde se despliega el nivel de actividad investigadora, adoptando la forma de:

- universidades puramente docentes de pregrado;

- docentes de pre y posgrado;

- docentes con investigación limitada a áreas específicas (por ejemplo, universidades politécnicas); y

- universidades de investigación con un enfoque comprensivo.

Esta última categoría puede generan una diferencia entre universidades que tienen facultad de medicina y las que no la tienen.

A su vez, el factor reputación se construye a partir de la posición de una universidad en rankings internacionales. Por lo tanto, dado el peso de la producción científica en muchos de estos rankings, el factor reputación se relaciona en una proporción significativa con el nivel de producción científica.

Schimank y Winners (2000) conceptualizan tres tipos de sistemas de educación superior que combinan investigación y docencia:

\section{Modelo francés}

Se caracteriza por una división del trabajo entre institutos de investigación (ejemplo: Institut National de la Santé et de la Recherche Médicale) y universidades, donde el foco está puesto en la docencia y la formación de capital humano (ejemplo: Sorbonne Université). En la práctica, en Francia, en las universidades se hace más investigación (65,3\%) que en los institutos públicos (52,3\%) (tabla 1), pero en el conjunto de países comparados, Francia muestra una menor participación relativa de las universidades en investigación.

\section{Modelo Humboldtiano}

Combina la docencia y la investigación en las universidades (ejemplo: Technische Universität München). Paradójicamente, las tres instituciones de investigación más productivas de Alemania son institutos públicos (Helmholtz Gemeinschaft, Max Planck Gesellschaft, Leibniz Gemeinschaft), lo que demuestra que el modelo Humboldtiano hoy no se cumple en Alemania.

\section{Modelo post-Humboldtiano}

Es el de los Estados Unidos de Norte América, en donde se diferencian las universidades que otorgan post grados y realizan investigación, respecto de las universidades de pregrado.

El anterior análisis pone de manifiesto que tanto el modelo francés, como el modelo Humboldtiano han perdido utilidad descriptiva.

En los países de América Latina, se da una variante del modelo post-Humboldtiano, pues las universidades que muestran una gran actividad investigadora realizan formación de pre y post grado. Es más, la Universidad Nacional Autónoma de México cuenta dentro de su estructura con 9 escuelas nacionales preparatorias (educación primaria), y 5 colegios de ciencias y humanidades (educación secundaria), así como en Colombia la Universidad del Rosario (fundada en 1653), cuenta con un colegio con niveles primario y secundario. Casos similares se dan en Chile y Perú.

En los EUA se aplica The Carnegie classification of institutions of higher education (Carnegie, 2018), que clasifica las instituciones de educación de nivel terciario de Estados Unidos. La primera clasificación fue publicada en 1973 y posteriormente actualizada nueve veces, siendo la más reciente la de 2018 con datos 2016-17. A partir de 2014 la clasificación es gestionada por la Indiana University School of Education. Para reconocer los diferentes tipos institucionales, la estructura de la clasificación se ha venido haciendo compleja con los años, dificultando la comparación intertemporal de las instituciones que la integran.

Según Carnegie las Doctoral universities son instituciones que al menos graduaron 20 doctores (PhD) o 30 doctores profesionales en un mínimo de 2 programas, y reportan al menos US\$5 millones en gastos en investigación en el año de actualización. Las universidades de esta categoría (tabla 3), a su vez son clasificadas en:

- R1: Doctoral universities: Very high research activity (131 institutions in 2016-17).

- R2: Doctoral universities: High research activity (135 institutions).

- D/PU: Doctoral/professional universities (132 institutions).

En 2016-17 son clasificadas de doctorado 398 universidades, lo que representa 9,2\% de las instituciones incluidas en la clasificación (tabla 3) (Carnegie, 2018). En la tabla 2 se aprecia que en Estados Unidos de Norte América 459 universidades registraron producciones superiores a 101 artículos en 2018 (58,5\% de 786). 
Tabla 3. Distribución de instituciones por clasificación, categoría y controlador

\begin{tabular}{|l|c|c|}
\hline \multirow{2}{*}{ Categoría } & \multicolumn{2}{c|}{ Número de instituciones } \\
\cline { 2 - 3 } & Públicas & Privadas non-profit \\
\hline Doctoral universities: Very high research activity & 94 & 37 \\
\hline Doctoral universities: High research activity & 91 & 43 \\
\hline Doctoral/Professional universities & 28 & 107 \\
\hline
\end{tabular}

Fuente: The Carnegie classification of institutions of higher education - Classification summary tables

Otros trabajos relevantes que muestran la evolución que han tenido las taxonomías universitarias son:

- Bonaccorsi y Daraio (2009): caracterizan el sistema universitario europeo usando microdatos.

- Daraio et al. (2011): caracterizan las universidades europeas basados en la información académica del Proyecto Aquameth.

- Vega-Tato (2009) propone una taxonomía para universidades de México.

- Muñoz y Blanco (2013), Reyes y Rosso (2013) y Améstica-Rivas et al. (2014) clasifican las universidades en Chile.

- Cruz-Castro et al. (2016) elaboran una tipología bidimensional construida sobre el compromiso de las universidades con las formas de actuar y la existencia de capacidades organizativas.

- Abankina et al. (2016) realizan una revisión de varias taxonomías universitarias y proponen una para las universidades rusas.

- Markuerkiaga et al. (2018) proponen una taxonomía de universidades emprendedoras.

- Casidy y Wymer (2018) desarrollan una taxonomía de los estudiantes universitarios en función de su orientación al logro y su sensibilidad al prestigio.

Las taxonomías y los modelos presentados tienen una limitada capacidad para generar una distinción entre universidades docentes, universidades docentes que hacen investigación y universidades de investigación. Concordamos con Marsh y Hattie (2002), Bonaccorsi y Daraio (2009), Daraio et al. (2011), y Coates et al. (2019) en que se hace necesario un modelo basado en información empírica.

\section{Rankings universitarios}

Las instituciones de educación superior, sean de carácter público o privado, compiten por estudiantes, profesores, recursos, donantes, y reconocimiento social (Piro; Sivertsen, 2016). Los estados de opinión respecto de las instituciones se construyen con base en atributos subjetivos. Los distintos actores de la sociedad aplican una percepción selectiva de la realidad, lo que genera de manera agregada una noción de reputación, que como es percibido, en muchos casos está sólidamente ganada y en otros no se condice con la realidad objetiva (Shin et al., 2011). Los rankings se han construido con arreglo a metodologías que miden la performance de una institución en relación con su función docente y de investigación, y con menor intensidad los impactos societales (Shin et al., 2011; Güleda; Umut, 2019). La evidencia demuestra que no está garantizado que las instituciones que avanzan en los rankings a las posiciones más altas han mejorado la calidad de sus procesos. Algunas instituciones trabajan sólo para mejorar su presencia en un ranking determinado. Una universidad que hace bien su tarea tiende a subir en los rankings, pero lo segundo siempre debiera ser consecuencia de lo primero y no al revés. Los rankings han demostrado ser una herramienta útil para la gestión institucional, aportando información empírica o permitiendo hacer benchmarking, lo que contribuye a mejorar los resultados en términos de efectividad y calidad (Erkkilä, 2013). Al igual que las taxonomías, los rankings universitarios enfrentan el desafío de ordenar instituciones con diferentes misiones, proyectos educativos, oferta académica de pre y post grado, capacidades investigativas, tamaños, recursos, historia, marcos regulatorios, control político-administrativo, y mecanismos de aseguramiento de la calidad (Moed, 2017; Pietrucha, 2018; Muga-Naredo; Sotomayor-Brulé, 2019).
Las taxonomías tienen una capacidad li- mitada para generar una distinción en- tre universidades docentes, universida- des docentes que hacen investigación y universidades de investigación. Se hace necesario un modelo basado en infor- mación empírica

Los rankings fueron inicialmente concebidos como un instrumento que permite medir la eficacia de una institución de educación superior, asumiendo que una universidad altamente ranqueada es más productiva, imparte docencia de alta calidad, realiza investigación de alto impacto, y hace una mayor contribución a la sociedad que las instituciones ranqueadas por debajo de ella (Vernon et al., 2018). Sin embargo, el fortalecimiento de una de las funciones sustantivas, como son docencia, investigación y extensión, pudieran generar un conflicto con el adecuado despliegue de las otras. Por ejemplo, como se observa en muchas universidades de Colombia, existe un conflicto entre investigación y proyección social. O que una institución de pequeño tamaño puede ser muy eficiente en los procesos formativos, manteniendo un bajo número de estudiantes por profesor, pero no ser muy eficiente en la producción de investigación. En el caso opuesto, una institución universitaria muy grande, puede ser muy eficiente en la generación de nuevo conocimiento, pero 
puede que la formación de los estudiantes de pregrado este básicamente entregada a profesores contratados a tiempo parcial y que no pertenecen a la plantilla de la universidad (Bornmann; Mutz, 2015).

Esta realidad pone de relieve la necesidad, al menos teórica, de medir cuándo se está en presencia de una institución de educación superior que alcanza la calidad. La estrecha relación entre calidad de la enseñanza e investigación ha sido ampliamente discutida, analizada y demostrada a través de los trabajos de Geschwind y Broström (2015), Pawar (2015), Mägi (2016), Rodríguez y Rubio (2016), Tight (2016), Gentile et al. (2017), Artés et al. (2017), Mayston (2017), Robert y Carlsen (2017), Shu-Ling-Huang y Shen (2017), y Gutiérrez-Rojas et al., (2019).
Los rankings fueron concebidos inicialmente como un instrumento que permite medir la eficacia de una institución de educación superior, asumiendo que una institución altamente ranqueada es más productiva, imparte docencia de alta calidad, realiza investigación de alto impacto, y hace una mayor contribución a la sociedad que las instituciones ranqueadas por debajo de ella (Shin et al., 2011)

Es difícil encontrar buenos indicadores de la calidad del aprendizaje, de la investigación y de la proyección social que representen los verdaderos resultados de las instituciones de educación superior, más aún si esta medición es de escala global. Los rankings realizan un esfuerzo para objetivar variables cualitativas mediante la identificación de indicadores cuantitativos que mejor representen las instituciones de educación superior que alcanzan la calidad. No todos los rankings se basan en información empírica. Algunos construyen indicadores a partir de opiniones recogidas mediante encuestas.

Los rankings universitarios se pueden clasificar básicamente como globales [World-Class Universities (Salmi, 2009); Global Higher Education (Erkkilä, 2013)] o nacionales. En este trabajo nos referiremos sólo a los primeros, analizando el peso que el componente investigación tiene en cada ranking, el alcance en término de número de instituciones cubiertas, el umbral de entrada para que una institución esté presente en el ranking, y si la metodología es consistente para todas las regiones o genera sub rankings con metodologías diferentes (tabla 4).

Explorar y medir la calidad de la investigación, como un atributo de la calidad de las instituciones de educación superior, es una preocupación de los investigadores desde la década de los 70. Si bien por años a nivel institucional y nacional se han utilizado los paneles de expertos (Husbands-Fealing et al., 2011), su aplicación difícilmente puede ser llevada a la escala de rankings globales. En este caso se debe recurrir a la ayuda de la cienciometría, la que mediante métodos cuantitativos caracteriza los resultados de la investigación (Nalimov; Mulcjenko, 1971). La unidad más básica de análisis en la cienciometría es la cita. El acto de citar la investigación de otro autor genera un enlace entre personas, ideas, revistas científicas, e instituciones, las cuales constituyen un campo empírico o red que puede ser analizada de modo cuantitativo (Mingers; Leydesdorff, 2015) para caracterizar una realidad que es cuantitativa y cualitativa (Vargas-Quesada et al., 2017). La cita establece una conexión en el tiempo entre un trabajo publicado de modo previo, las referencias en él incluidas, y las citas a él otorgadas (Mingers; Leydesdorff, 2015).

Paradójicamente, el tamaño de la producción científica no es un indicador de calidad, sino sólo de intensidad investigadora (Claassen, 2015). Medir el tamaño favorece a las instituciones grandes. Se requiere un conjunto de indicadores, que sin ser ninguno un indicador de calidad, a partir de una lectura combinada de los mismos se genere una noción de calidad de la producción científica de un actor determinado.
El acto de citar genera un enlace entre personas, ideas, revistas científicas, e instituciones, las cuales constituyen una red que puede ser analizada de modo cuantitativo y cualitativo (Mingers; Leydesdorff, 2015)

Un ranking global de instituciones de educación superior debiera caracterizarse por:

- Transparencia, relevancia, y validez de los datos (Berlin principles on ranking of higher education institutions, Unesco, 2016).

- Usar un conjunto de indicadores que caractericen la calidad de la investigación.

- Usar sólo indicadores aplicables globalmente (Erkkilä, 2013).

- Los indicadores debieran ser generados por terceros, independientes de las instituciones ranqueadas.

- Los datos, idealmente, debieran ser recopilados por agentes diferentes de los que construyen los indicadores.

- Basarse en información objetiva, no en percepciones públicas obtenidas por encuestas (SCImago, 2019).

- Que la ponderación de los indicadores esté basada en opiniones de expertos independientes de la institución que genera el ranking (SCImago, 2019).

- Establecer unos umbrales mínimos de entrada. El tamaño importa, pero no lo es todo. Por debajo de un cierto tamaño los indicadores independientes de los tamaños son inoperantes (SCImago, 2019).

Es deseable generar indicadores corregidos por tamaño institucional, pero ello entraña grandes riesgos. Algunos ejemplos fallidos son: si se dividiera el número de artículos científicos publicados en un año por el número de profesores a jornada completa equivalente (FTE, full time equivalent), la duda para cualquier oficina de análisis institucional es definir que es un FTE, y el problema se vuelve inmanejable cuando se aplica para todo el mundo. Si el número de trabajos se 
dividiera por el stock de investigadores activos $\left(\mathrm{STP}^{3}\right)$, lo que se obtendría es la tasa de coautoría y no un indicador de productividad. Además, si los trabajos tienen participación de profesores-investigadores y estudiantes, especialmente de post grado o colaboración con autores de otras instituciones no se puede calcular la producción per cápita. Muchos rankings miden el número de trabajos publicados, citas por académico y monto de recursos externos. Si se dividiera el número de citas por artículo publicado, o por investigador activo, no se estaría reconociendo que diferentes categorías temáticas tienen diferentes pautas o intensidades de citación. Alguna de las ratios como las antes indicadas favorecen a las universidades intensivas en STEM ${ }^{4}$ y perjudican a las universidades que buena parte de su producción se concentra en ciencias sociales y humanidades (tabla 4).

Tabla 4. Comparación de los principales rankings universitarios globales

\begin{tabular}{|c|c|c|c|c|c|c|c|c|}
\hline Ranking & $\begin{array}{l}\text { Institucio- } \\
\text { nes uni- } \\
\text { versitarias } \\
\text { participan- } \\
\quad \text { tes }\end{array}$ & $\begin{array}{l}\text { \# de indi- } \\
\text { cadores de } \\
\text { investiga- } \\
\text { ción }\end{array}$ & $\begin{array}{l}\text { \# mínimo de } \\
\text { documentos } \\
\text { anuales de } \\
\text { ingreso al } \\
\text { ranking }\end{array}$ & $\begin{array}{l}\text { Tiene } \\
\text { publici- } \\
\text { dad }\end{array}$ & $\begin{array}{l}\text { Fuente } \\
\text { de datos } \\
\text { científi- } \\
\text { cos }\end{array}$ & $\begin{array}{l}\text { Cambia la } \\
\text { meto- } \\
\text { dología } \\
\text { según } \\
\text { regiones }\end{array}$ & Versión & Web \\
\hline $\begin{array}{l}\text { SCImago Institutions } \\
\text { Rankings (SIR) }\end{array}$ & 3.468 & 11 & $\begin{array}{l}100 \text { docu- } \\
\text { mentos en el } \\
\text { último año }\end{array}$ & No & Scopus & No & 2019 & https://www.scimagoir.com \\
\hline $\begin{array}{l}\text { QS World University } \\
\text { Rankings }\end{array}$ & 1.002 & 1 & no define & Sí & Scopus & Sí & 2020 & $\begin{array}{l}\text { https://www.topuniversities. } \\
\text { com/university-rankings/ } \\
\text { world-university-rankings/2020 }\end{array}$ \\
\hline $\begin{array}{l}\text { Shanghai Ranking's } \\
\text { (Academic Ranking of } \\
\text { World Universities) }\end{array}$ & 1.000 & 3 & no define & No & WoS & No & 2019 & $\begin{array}{l}\text { http://www.shanghairanking. } \\
\text { com }\end{array}$ \\
\hline $\begin{array}{l}\text { The Time Higher } \\
\text { Education World } \\
\text { University Ranking }\end{array}$ & 1.250 & 4 & $\begin{array}{l}1.000 \text { docu- } \\
\text { mentos en } 5 \\
\text { años }\end{array}$ & Sí & Scopus & Sí & 2019 & $\begin{array}{l}\text { https://www. } \\
\text { timeshighereducation. } \\
\text { com/world-university- } \\
\text { rankings/2019/world-ranking }\end{array}$ \\
\hline $\begin{array}{l}\text { CWTS Leiden Ranking } \\
\text { (CWTS) }\end{array}$ & 1.000 & 10 & no define & No & Scopus & No & 2019 & $\begin{array}{l}\text { https://www.leidenranking. } \\
\text { com }\end{array}$ \\
\hline $\begin{array}{l}\text { Center for World } \\
\text { University Rankings } \\
\text { (CWUR) }\end{array}$ & 2.000 & 6 & no define & No & WoS & No & $2019-20$ & https://cwur.org \\
\hline $\begin{array}{l}\text { The U.S. News } \\
\text { Rankings-Best Global } \\
\text { Universities }\end{array}$ & 1.250 & 13 & no define & Sí & WoS & No & 2019 & $\begin{array}{l}\text { https://www.usnews.com/ } \\
\text { education/best-global- } \\
\text { universities }\end{array}$ \\
\hline
\end{tabular}

El análisis de los rankings presentados en la tabla 4 muestra la siguiente evidencia:

- Existe una alta convergencia en los rankings de incluir un mínimo de 1.000 universidades. Es interesante observar que sólo el SCImago Institutions Rankings (SIR) y The Time Higher Education World University Ranking introducen limites de tamaños de producción científica para la inclusión de nuevas universidades, el primero asociable a instituciones que pasan de universidades docentes a universidades docentes que hacen investigación, y el segundo ligado universidades que pasan de universidades docentes que hacen investigación a universidades de investigación. El más inclusivo es el ranking de SClmago.

- Existe una alta variación en el número de indicadores cienciométricos, pero lo más preocupante es que algunos rankings usan indicadores de investigación metodológicamente muy cuestionables (Dobrota; Dobrota, 2016).

- Otro elemento a considerar es que algunos rankings presentan metodologías diferentes del general cuando generan rankings por regiones geográficas, lo cual hace que una misma institución aparezca en posiciones relativas diferentes según la versión del ranking que se consulte.

- Aceptar publicidad en los rankings genera un posible conflicto de interés.

Pese a todas estas potenciales inconsistencias, los rankings globales son una aproximación a un modelo basado en información empírica.

\section{Información académica}

Tradicionalmente la información académica es generada por unidades de inteligencia competitiva, denominadas unidades de análisis institucional (Cifuentes-Madrid et al., 2015). Estas unidades generan información académica para asegurar la calidad de los procesos (Tavares et al., 2016), de los programas, dar cuenta pública de los resultados y disponer de un conjunto de indicadores estratégicos propios y de la competencia, así como generar información estratégica para la toma de decisiones. Sin embargo, el análisis de los resultados de la actividad investigativa, por su naturaleza especializada recae en unidades de análisis cienciométrico situadas en vicerrectorías de investigación (Santelices, 2010) y en algunos contados casos en direcciones de biblioteca. 
El nivel de los análisis de la actividad investigadora se centra en el recuento de la producción indexada en WoS o Scopus, el cuartil de la revista de publicación, y el análisis de redes de colaboración. Muy pocas de estas unidades cuentan con capacidad de acceder a indicadores que caractericen detalladamente la producción, y mucho menos los indicadores de performance, impacto y excelencia. Para ello recurren a:

- Scopus para analizar el índice h de los autores o el SNIP de una revista;

- SJR y JCR para conocer los cuartiles de las revistas;

- rankings: el SCImago Institutions Rankings:

https://www.scimagoir.com

- de modo específico a informes de alcance regional, generados con la misma metodología internacional, como SIR Iber: https://www.elprofesionaldelainformacion.com/informes_scimago_epi/SIR_lber_2019.html

que provee indicadores como: Impacto Normalizado Liderado (NIwL), Proporción de producción en Excelencia 10 $(\mathrm{Exc})$, Proporción de producción Liderada (L), Proporción de producción en Excelencia 10 Liderada (EwL), Scientific Talent Pool (STP), Conocimiento Innovador (número de publicaciones de una institución citadas en patentes) (IK), e Impacto Tecnológico (proporción de publicaciones de una institución citadas en patentes) (TI), entre otros indicadores que caracterizan los resultados de la actividad investigativa (Frenken et al., 2017; De-Moya-Anegón et al., 2019);

- rankings como el de la Universidad de Leiden CWTS.

Este último sólo cubre 1.000 universidades, todas clasificables como universidades de investigación. Sólo SCImago Institutions Rankings, que cubre más de 3.400 entidades de educación superior, ofrece indicadores para universidades de investigación y universidades docentes que hacen investigación.

La caracterización de la producción científica debe equilibrar los indicadores que dependen del tamaño de la producción, con aquellos que describen performance, impacto y excelencia. El análisis de la información empírica demuestra que algunas instituciones de todos los países analizados, de todos los tamaños, presentan resultados de impacto que están descendidos de la media del mundo, o que sus resultados de Excelencia 10 o Excelencia 1 no alcanzan ni el $10 \%$ ni el $1 \%$, respectivamente, esperado, lo cual advierte que ser grande no es lo mismo que ser bueno.

Una segunda observación empírica es que algunas universidades logran indicadores de Impacto o Excelencia muy altos, pero cuando se filtran por producción liderada, la distancia entre ambos valores es enorme, quedando los indicadores bajo los niveles esperados. Eso habla de que existen universidades que son capaces de generar resultados de calidad de forma autónoma, y otras que lo consiguen mediante la asociación con instituciones o investigadores que aportan producción de calidad. Una universidad que lidera su producción define la agenda de temas a investigar, demuestra capacidad de obtener financiamiento para su investigación y que cuenta con claustros doctorales con capacidad de formar doctores que pueden realizar investigación original de modo autónomo.

¿Cómo una comunidad universitaria puede formar un juicio crítico sobre sus resultados de investigación? ¿Cómo el mundo o la comunidad internacional y nacional se dan cuenta de ese nivel de logro? La primera pregunta, tiene dos respuestas:

- la primera es asistemática, y consiste en la auto percepción que cada investigador tiene de su propio trabajo y el de sus colegas, especialmente de los temáticamente próximos, donde cuenta con categorías de análisis para juzgar los resultados;

- la segunda es a partir de la información que la propia universidad pueda generar respecto de sus resultados. La evidencia muestra que conformar ese juicio de valor es complejo y está expuesto a los riesgos propios de los juicios particulares. Siendo eso difícil, comunicar al mundo los resultados de una universidad es complejo (Shi; Coates, 2018).

La respuesta a la segunda pregunta es más compleja aún, pues ese estado de opinión se forma a partir de las percepciones de los actuales estudiantes y de los egresados, de los actuales y anteriores académicos, de la opinión de los empleadores, de las noticias negativas y positivas que circulan en prensa y redes sociales, de la evidencia disponible en los sistemas públicos de información para postulantes, del nivel de acreditación obtenido, especialmente institucional (Stura et al., 2019), de la posición de la institución en los rankings, especialmente los globales, entre muchas otras variables. Lo que queda en evidencia es lo altamente crítico que resulta ser para una universidad que lo que se difunda sea información fidedigna, consistente y relevante, así como lo importante que es el uso que se haga de dicha información.

Presentamos a continuación tres casos actuales. Uno de carácter institucional, uno de diseño de un sistema nacional, y uno relativo al uso que se da a las declaraciones internacionales y el grado de adhesión de los actores institucionales.

Se presenta el caso de la Universidad de la Costa (CUC), situada en Barranquilla, Colombia. En 2013 produjo 8 artículos publicados en revistas Scopus y ocupó la posición número 77 del conjunto de universidades colombianas que hacen investigación. En 2018 publicó 296 documentos en la misma fuente, ocupando la décima posición de producción en el conjunto de universidades del país. Un salto enorme que las demás universidades del país observan con estupor. Pero si a esa evidencia de fácil acceso se agrega que el Impacto Normalizado fue de 0,92 (situándola novena del país), que el Impacto Normalizado liderado fue de 0,86 (tercera del país), y que el 5,88\% de su producción alcanzó la Excelencia 10 (tercera del país) (todos los indicadores referidos a 2018), instala la idea de que el crecimiento en cantidad ha ido acompañado de la calidad, todo lo cual pone a la CUC en una posición relevante en el contexto nacional. 
En América Latina se dan otros casos interesantes de crecimiento cuantitativo aparejado con mejoras en los indicadores cualitativos, como la Pontificia Universidad Católica de Valparaíso y la Universidad Andrés Bello, ambas en Chile, la Universidad Peruana de Ciencias Aplicadas, o la Universidad Panamericana en México.

En el Cono Sur de América Latina existe un país en donde el organismo responsable de las universidades construye por mandato legal un ranking de instituciones de educación superior. Lo curioso es que este organismo también debe acreditar instituciones, sobre las cuales ya emitió un juicio previo al generar un ranking. La segunda curiosidad son las ventanas de observación de algunos indicadores que utilizan ventanas de 10 años. Con ventanas tan amplias se consagra la inercia estructural. En ese país se está impidiendo que universidades como la antes ejemplificada, que han experimentado cambios muy dinámicos, sean visualizadas adecuadamente en dicho ranking público nacional, reflejando su nueva condición. Andreani et al. (2019) dan cuenta de otros aspectos sobrios en los procesos de acreditación.

Una tercera situación es cuando proyectos de agregadores a texto completo de revistas científicas internacionales piden a las instituciones editoras de las revistas, que son mayoritariamente universidades, que firmen declaraciones como DORA, que obliga a los editores a renunciar al uso de indicadores como el Impact Factor (IF) de los JCR para clasificar sus revistas dentro de una categoría temática. Las declaraciones como DORA-San Francisco (2012) o Leiden (2015) hacen un llamado a las agencias de valoración de investigadores y asignación de fondos de investigación a no medir el trabajo de los investigadores a partir del impacto esperado de la revista de publicación (IF) (Waltman, 2016), sino por el impacto observado y otros indicadores derivados de la medición de los resultados particulares alcanzados por los artículos publicados. Algunos organismos nacionales de ciencia y tecnología, así como agencias nacionales de acreditación, también están convencidos de que un investigador, un claustro de investigadores o una institución pueden ser medidos por el Impact Factor de la revista de publicación, lo cual es claramente un error metodológico.

Estos casos demuestran que la información académica no sólo tiene problemas de generación, sino de acceso y sobre todo de uso posterior, pues las metodologías usadas no siempre permiten generar conclusiones válidas.

\section{Tránsito de una universidad docente a una de investigación}

¿Dónde se establece el límite entre universidad docente, docente que hace investigación y universidad de investigación? No existe una definición taxativa de cuál es esa frontera de tamaño, calidad y autonomía investigativa. Usando la evidencia presentada en las tablas 1 y 2, y la taxonomía de Carnegie, se diría que es una universidad de investigación una institución que produce entre 1.000 y 2.000 documentos por año y gradúa 20 o más doctores (PhD) por año. Esta clasificación no dice nada de la calidad, porque en el caso de Estados Unidos de Norte América se da por hecho. Esas fronteras de tamaño aplican a Estados Unidos de Norte América. ¿Existe una única frontera para todo el mundo? $\mathrm{O}$ debiéramos tener unas fronteras relativas a las características de los sistemas universitarios nacionales, regionales (UE, Asia, América Latina, OCDE, Briccs). La evidencia presentada en las tablas 1 y 2 insinúa que parece no justificarse.

Quizá el mayor desafío no es identificar las universidades de investigación de las otras, sino la diferenciación entre una universidad docente y una universidad docente que hace investigación. La tabla 2 muestra que en todos los países observados hay un alto número de instituciones con una producción menor de 100 documentos anuales. Son un grupo dinámico, porque los tamaños son pequeños, y los crecimientos se dan con mayor facilidad. La evidencia indica que las universidades con menos de 100 documentos por año no ofrecen programas de doctorado, y si lo hacen, son pocos y no acreditados. Tal es el caso de Brasil, México, Argentina, Chile y Colombia, países en los que existen mecanismos oficiales de acreditación de programas de doctorado.

Un segundo elemento a considerar es el umbral mínimo de entrada a los rankings de universidades con foco en investigación. Sólo dos rankings declaran este límite:

- SClmago Institutions Rankings: establece ese nivel en 100 artículos en el último año, consideran una ventana de observación de 5 años;

- The Time Higher Education World University Ranking: lo fija en 1.000 documentos en 5 años.

Una tercera mirada es correlacionar universidades acreditadas y tamaños de producción científica (Shehatta; Mahmood, 2016). En el caso de Colombia ese umbral es 150 artículos científicos por año, y en el caso de Chile, la última universidad acreditada en investigación por la Comisión Nacional de Acreditación es la Universidad de Magallanes (la más austral del mundo), con 154 documentos en 2018.

Por lo tanto, una universidad docente que hace investigación como mínimo produce 100 artículos por año, siendo deseable que ese umbral de tamaño esté por encima de los 150 artículos anuales. Una universidad de investigación produce entre 1.000 y 2.000 artículos por año y gradua al menos 20 doctores por año. En ambos casos, las instituciones deben alcanzar unos indicadores de performance, impacto y excelencia, ambos liderados,
Una universidad docente que hace investigación como mínimo produce 100 artículos por año, siendo deseable que ese umbral de tamaño esté por encima de los 150 artículos anuales 
que demuestren que en la universidad existe un claustro de profesores en condiciones de hacer investigación original de forma autónoma, con un nivel de resultados equivalentes a los de sus colegas en el mundo.
Una universidad de investigación produce entre 1.000 y 2.000 artículos por año y gradua al menos 20 doctores por año

\section{Conflictos de interés}

El autor es miembro de SCImago Research Group, la empresa consultora que genera el SCImago Institutions Rankings. El autor fungió como editor invitado de este número temático dedicado a la información académica pero no participó en la evaluación de este artículo.

\section{Notas}

1. Investigación participada: son los resultados de investigación en donde al menos un autor tiene filiación institucional perteneciente a un sector definido. Dado el fenómeno de la coautoría en colaboración interinstitucional, un trabajo puede ser contabilizado en más de un sector.

2. Sectorización de la producción científica: los métodos de normalización utilizados por SCImago incluyen una amplia desambiguación de los nombres de las instituciones a través de la afiliación institucional de los documentos incluidos en Scopus. La tarea de identificación incluye la consolidación o separación de instituciones, así como el tratamiento de los cambios de nombre. El objetivo de la normalización es doble, definir e identificar las instituciones mediante la elaboración de una lista de instituciones de investigación en las que cada institución está correctamente identificada y definida, y atribuir publicaciones y citas a cada institución. Para lograrlo, SCImago tiene en cuenta la afiliación institucional de cada autor en el campo "afiliación" de la base de datos Scopus. Se aplica un sistema híbrido que comprende elementos manuales y automáticos para asignar afiliaciones a una o más instituciones, según proceda. Una vez identificadas correctamente las instituciones, se agrupan en cinco sectores: educación superior, gobierno, salud, privado y otros (instituciones privadas sin fines de lucro).

3. STP (scientific talent pool): representa el número de autores diferentes de una misma institución que han participado en el total de trabajos publicados. Refleja el número de investigadores activos con los que cuenta la institución y, en consecuencia, el tamaño de su fuerza de trabajo (De-Moya-Anegón et al., 2019).

4. STEM (science, technology, engineering, mathematics): acrónimo para referir las áreas de conocimiento ciencia, tecnología, ingeniería y matemáticas, por sus iniciales en inglés.

\section{Referencias bibliográficas}

Abankina, Irina; Aleskerov, Fuad; Belousova, Veronika; Gokhberg, Leonid; Kiselgof, Sofya; Petrushchenko, Vsevolod; Shvydun, Sergey; Zinkovsky, Kirill (2016). "From equality to diversity: Classifying Russian universities in a performance oriented system". Technological forecasting and social change, v. 103, pp. 228-239.

https://doi.org/10.1016/j.techfore.2015.10.007

Améstica-Rivas, Luis; Gaete-Feres, Héctor; Llinàs-Audet, Xavier (2014). "Segmentación y clasificación de las universidades en Chile: desventajas de inicio y efectos de las políticas públicas de financiamiento". Ingeniare. Revista chilena de ingeniería, v. 22, n. 3, pp. 384-397.

https://doi.org/10.4067/S0718-33052014000300009

Andreani, Martino; Russo, Daniel; Salini, Silvia; Turri, Mateo (2019). "Shadows over accreditation in higher education: Some quantitative evidence". Higher education, online first.

https://doi.org/10.1007/s10734-019-00432-1

Artés, Joaquín; Pedraja-Chaparro, Francisco; Salinas-Jiménez, María-del-Mar (2017). "Research performance and teaching quality in the Spanish higher education system: Evidence from a medium-sized university". Research policy, v. 46, n. 1, pp. 19-29.

https://doi.org/10.1016/j.respol.2016.10.003

ARWU (2019). Academic Ranking of World Universities.

http://www.arwu.org

Bonaccorsi, Andrea; Daraio, Cinzia (2009). "Characterizing the European university system: A preliminary classification using census microdata". Science and public policy, v. 36, n. 10, pp. 763-775.

https://doi.org/10.3152/030234209X475245

Bornmann, Lutz; Mutz, Rüdiger (2015). "How well does a university perform in comparison with its peers? The use of odds, and odds ratios, for the comparison of institutional citation impact using the Leiden Rankings". Journal of the Association for Information Science and Technology, v. 66, n. 12, pp. 2711-2713.

https://doi.org/10.1002/asi.23451

Brunner, José-Joaquín (2007). Educación superior en Iberoamérica. Informe 2007. Santiago de Chile: Centro Interuniversitario de Desarrollo. 317 p. ISBN: 9789567106509

https://cinda.cl/publicacion_archivos/educacion-superior-en-iberoamerica-informe-2007 
Brunner, José-Joaquín (2009). Tipología y características de las universidades chilenas. Documento para comentarios. Centro de Políticas Comparadas en Educación, Universidad Diego Portales.

http://200.6.99.248/ bru487cl/files/Tipol\%26Caract_080209.pdf

Carnegie Foundation for the Advancement of Teaching (2018). The Carnegie Classification of Institutions of Higher Education. 2018 update. Facts \& figures. Descriptive highlights.

http://carnegieclassifications.iu.edu/downloads/CCIHE2018-FactsFigures.pdf

Casidy, Riza; Wymer, Walter (2018). "A taxonomy of prestige-seeking university students: Strategic insights for higher education". Journal of strategic marketing, v. 26, n. 2, pp. 140-155.

https://doi.org/10.1080/0965254X.2016.1182573

Cifuentes-Madrid, Jairo H.; Landoni-Couture, Pablo; Llinàs-Audet, Xavier (eds.) (2015). Strategic management of universities in the Ibero-American region. A comparative perspective. Heidelberg, Springer, 146 p. ISBN: 9783319146843 https://doi.org/10.1007/978-3-319-14684-3

Claassen, Christopher (2015). “Measuring university quality". Scientometrics, v. 104, n. 3, pp. 793-807.

https://doi.org/10.1007/s11192-015-1584-8

Coates, Hamish; Liu, Lu; Shi, Jinghuan (2019). "Evaluating complex higher education systems". International journal of Chinese education, v. 8, n. 1, pp. 1-5.

https://doi.org/10.1163/22125868-12340101

Cruz-Castro, Laura; Benítez-Amado, Alberto; Sanz-Menéndez, Luis (2016). "The proof of the pudding: University responses to the European research council". Research evaluation, v. 25, n. 4, pp. 358-370.

https://doi.org/10.1093/reseval/rvw015

Daraio, Cinzia; Bonaccorsi, Andrea; Geuna, Aldo; Lepori, Benedetto; Bach, Laurent; Bogetoft, Peter; Cardoso, Margarida F.; Castro-Martínez, Elena; Crespi, Gustavo; Fernández-de-Lucio, Ignacio; Fried, Harold; García-Aracil, Adela; Inzelt, Annamaria; Jongbloed, Ben; Kempkes, Gerhard; Llerena, Patrick; Matt, Mireille; Olivares, María; Pohl, Carsten; Raty, Tarmo; Rosa, Maria J.; Sarrico, Cláudia S.; Simar, Léopold; Slipersaeter, Stig; Teixeira, Pedro N.; Eeckaut, Philippe-Vanden (2011). "The European university landscape: A micro characterization based on evidence from the Aquameth project". Research policy, v. 40, n. 1, pp. 148-164.

https://doi.org/10.1016/j.respol.2010.10.009

Daraio, Cinzia; Bonaccorsi, Andrea; Simar, Léopold (2015). "Rankings and university performance: A conditional multidimensional approach". European journal of operational research, v. 244, n. 3, art. no. 12774, pp. 918-930.

https://doi.org/10.1016/j.ejor.2015.02.005

De-Moya-Anegón, Félix; Herrán-Páez, Estefanía; Bustos-González, Atilio; Corera-Álvarez, Elena; Tibaná-Herrera, Gerardo; Rivadeneyra, Federico (2019). Ranking Iberoamericano de instituciones de educación superior 2019 (SIR Iber). Granada: El profesional de la información. ISBN: 9788412023916

https://doi.org/10.3145/sir-iber-2019

De-Moya-Anegón, Félix; López-Illescas, Carmen; Moed, Henk F. (2014). “How to interpret the position of private sector institutions in bibliometric rankings of research institutions". Scientometrics, v. 98, n. 1, pp. 283-298.

https://doi.org/10.1007/s11192-013-1087-4

Dobrota, Milan; Dobrota, Marina (2016). "ARWU ranking uncertainty and sensitivity: What if the award factor was excluded?". Journal of the Association for Information Science and Technology, v. 67, n. 2, pp. 480-482.

https://doi.org/10.1002/asi.23527

DORA. San Francisco Declaration on Research Assessment (2012).

https://sfdora.org/read

Erkkilä, Tero (ed.) (2013). Global university rankings. Challengues for European higher education. Hampshire, UK.; Palgrave MacMillan. 255 p. ISBN: 9781137296870

https://doi.org/10.1057/9781137296870

Frenken, Koen; Heimeriks, Gaston J.; Hoekman, Jarno (2017). "What drives university research performance? An analysis using the CWTS Leiden Ranking data". Journal of informetrics, v. 11, n. 3, pp. 859-872.

https://doi.org/10.1016/j.joi.2017.06.006

Gentile, James; Brenner, Kerry; Stephens, Amy (eds.) (2017). Undergraduate research experiences for STEM students: Successes, challenges, and opportunities. Washington, DC: The National Academies Press. ISBN: 9780309452830 https://doi.org/10.17226/24622

Geschwind, Lars; Broström, Anders (2015). "Managing the teaching-research nexus: ideals and practice in research-oriented universities". Higher education research and development, v. 34, n. 1, pp. 60-73.

https://doi.org/10.1080/07294360.2014.934332 
González-Albo, Borja; Aparicio, Javier; Moreno, Luz; Bordons, María (2016). “Los sectores institucionales en la producción científica española de difusión internacional”. Revista española de documentación científica, v. 39, n. 1, art. e115. https://doi.org/10.3989/redc.2016.1.1260

Güleda, Doğan; Umut, Al (2019). "Is it possible to rank universities using fewer indicators? A study on five international university rankings". Aslib journal of information management, v. 71, n. 1, pp. 18-37. https://doi.org/10.1108/AJIM-05-2018-0118

Gutiérrez-Rojas, Iván R.; Peralta-Benítez, Hipólito; Fuentes-González, Homero C. (2019). “Integración de la investigación y la enseñanza en las universidades médicas”. Educación medica, v. 20, n. 1, pp. 49-54.

https://doi.org/10.1016/j.edumed.2018.07.007

Husbands-Fealing, Kane; Lane, Julia I.; Marbuerger III, John H.; Shipp, Stephanie S. (2011). The science of science policy. Stanford, California: Stanford Business Books, 385 pp. ISBN: 9780804770781

https://doi.org/10.1111/j.1541-1338.2011.00523.x

Leiden manifesto for research metrics (2015).

http://www.leidenmanifesto.org

Markuerkiaga, Leire; Igartua, Juan-Ignacio; Errasti, Nekane (2018). “A performance-based taxonomy of entrepreneurial universities". International journal of technology management, v. 77, n. 1-3, pp. 57-85.

https://doi.org/10.1504/IJTM.2018.091713

Marsh, Herbert W.; Hattie, John (2002). "The relation between research productivity and teaching effectiveness: Complementary, antagonistic, or independent constructs?". Journal of higher education, v. 73, n. 5, pp. 603-641. https://doi.org/10.1080/00221546.2002.11777170

Mayston, David J. (2017). "Convexity, quality and efficiency in education”. Journal of the operational research society, v. 68, n. 4, pp. 446-455.

https://doi.org/10.1057/jors.2015.91

Mingers, John; Leydesdorff, Loet (2015). "A review of theory and practice in Scientometrics". European journal of operational research, v. 246, n. 1, pp. 1-19.

https://doi.org/10.1016/j.ejor.2015.04.002

Moed, Henk F. (2017). "A critical comparative analysis of five world university rankings". Scientometrics, v. 110, n. 2, pp. $967-990$. https://doi.org/10.1007/s11192-016-2212-y

Muga-Naredo, Alfonso; Sotomayor-Brulé, Alejandro (2019). "Sistemas de evaluación de calidad y control político-administrativo". Revista educación superior y sociedad, v. 22, pp. 111-130.

http://www.iesalc.unesco.org/ess/index.php/ess3/article/view/35/35

Muñoz, Miguel; Blanco, Christian (2013). “Una taxonomía de las universidades chilenas". Calidad en la educación, n. 38, pp. 181-213.

https://doi.org/10.4067/S0718-45652013000100005

Nalimov, Vasily; Mulcjenko, B. (1971). Information model of the process of the development of science. Washington DC: Foreign Technology Division.

http://garfield.library.upenn.edu/nalimovmeasurementofscience/chapter1.pdf

OCDE; SCImago Research Group (2016). Compendium of bibliometric science indicators. Paris: OECD.

http://oe.cd/scientometrics

Patra, Swapan-Kumar; Muchie, Mammo (2018). "Research and innovation in South African universities: From the triple helix's perspective". Scientometrics, v. 116, n. 1, pp. 51-76.

https://doi.org/10.1007/s11192-018-2764-0

Pawar, Badrinarayan-Shankar (2015). "Enhancing research-teaching link in organizational behavior: Illustration through an actual example". The international journal of management education, v. 13, n. 3, pp. 326-336.

https://doi.org/10.1016/j.ijme.2015.10.005

Pietrucha, Jacek (2018). "Country-specific determinants of world university rankings". Scientometrics, v. 114, n 3, pp. 1129-1139.

https://doi.org/10.1007/s11192-017-2634-1

Piro, Fredrik-Niclas; Sivertsen, Gunnar (2016). "How can differences in international university rankings be explained?". Scientometrics, v. 109, n. 3, pp. 2263-2278.

https://doi.org/10.1007/s11192-016-2056-5 
Reyes, Claudia; Rosso, Pedro-Pablo (2013). “Una nueva clasificación de las universidades chilenas”. En: Santelices, María-Verónica; Ugarte, J. J.; Salmi, Jamil. Clasificación de instituciones de educación superior. Ministerio de Educación de Chile, pp. 135-151.

Robert, Jenay; Carlsen, William S. (2017). "Teaching and research at a large university: Case studies of science professors". Journal of research in science teaching, v. 54, n. 7, pp. 937-960.

https://doi.org/10.1002/tea.21392

Rodríguez, Rosa; Rubio-Irigoyen, Gonzalo (2016). "Teaching quality and academic research". International review of economics education, v. 23, pp. 10-27.

https://doi.org/10.1016/j.iree.2016.06.003

Salmi, Jamil (2009). The challenge of establishing world-class universities. Washington DC: The International Bank for Reconstruction and Development / The World Bank, $136 \mathrm{pp}$.

https://doi.org/10.1596/978-0-8213-7865-6

Santelices, Bernabé (ed.) (2010). El rol de las universidades en el desarrollo científico y tecnológico. Educación Superior en Iberoamérica. Informe 2010. Santiago de Chile: Centro Interuniversitario de Desarrollo, 241 pp. ISBN: 9789567106554 https://cutt.ly/IwnDGgv

Schimank, Uwe; Winners, Markus (2000). "Beyond Humboldt? The relationship between teaching and research in European university systems". Science \& public policy, v. 27, n. 6, pp. 397-408.

https://doi.org/10.3152/147154300781781733

Shehatta, Ibrahim; Mahmood, Khalid (2016). "Correlation among top 100 universities in the major six global rankings: policy implications". Scientometrics, v. 109, n. 2, pp. 1231-1254.

https://doi.org/10.1007/s11192-016-2065-4

Shi, Jinghuan; Coates, Hamish (2018). "Constructing higher education evaluation systems for the global era". International journal of Chinese education, v. 7, n. 2, pp. 175-180.

https://doi.org/10.1163/22125868-12340095

Shin, Jung-Cheol; Toutkoushian, Robert K.; Teichler, Ulrich (eds.) (2011). University rankings. Theoretical basis, methodology and impacts on global higher education. London: Springer, 271 pp. ISBN: 9789400711167 https://doi.org/10.1007/978-94-007-1116-7

Shu-Ling-Huang, Sheila; Shen, Shuo-Pin (2017). "Relationships among academic ranks, numbers of funded projects, and research productivity of university faculty: The case of a private medical university". Journal of research in education sciences, v. 62, n. 4, pp. 89-115.

https://doi.org/10.6209/JORIES.2017.62(4).04

Stura, Ilaria; Gentile, Tiziana; Migliaretti, Giuseppe; Vesce, Enrica (2019). "Accreditation in higher education: Does disciplinary matter?". Studies in educational evaluation, v. 63, pp. 41-47.

https://doi.org/10.1016/j.stueduc.2019.07.004

Tavares, Orlanda; Sin, Cristina; Amaral, Alberto (2016). "Internal quality assurance systems in Portugal: What their strengths and weaknesses reveal". Assessment and evaluation in higher education, v. 41, n. 7, pp. 1049-1064.

https://doi.org/10.1080/02602938.2015.1064515

Unesco (2016). Berlin principles on ranking of higher education institutions. Bucharest: Unesco (Cepes); Whashington D.C.: Institute for Higher Education Policy.

https://www.che.de/downloads/Berlin_Principles_IREG_534.pdf

Vargas-Quesada, Benjamín; Bustos-González, Atilio; De-Moya-Anegón, Félix (2017). "SCImago institutions rankings: The most comprehensive ranking approach to the world of research institutions". In: Cantu-Ortiz, Francisco J. (ed.). Research analytics: Boosting university productivity and competitiveness through scientometrics, pp. 147-160. https://doi.org/10.1201/9781315155890

Vega-Tato, Griselda Z. (2009). "Poniendo orden a las instituciones particulares de educación superior en México: una taxonomía aplicada a su complejidad y diversidad". Revista de la educación superior, v. 38, n. 150, pp. 37-60. http://www.scielo.org.mx/scielo.php?script=sci_arttext\&pid=S0185-27602009000200003

Vernon, Mario M.; Balas, E. Andrew; Momani, Shaher (2018). "Are university rankings useful to improve research? A systematic review". PLoS one, v. 13, n. 3, art. e0193762.

https://doi.org/10.1371/journal.pone.0193762

Waltman, Ludo (2016). "A review of the literature on citation impact indicators". Journal of informetrics, v. 10, n. 2, pp. $365-391$. https://doi.org/10.1016/j.joi.2016.02.007 


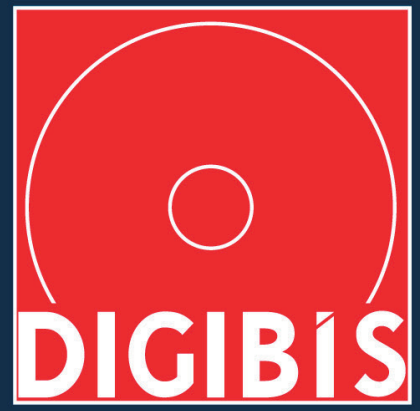

Nuestra vocación: contribuir a que el patrimonio cultural español sea preservado digitalmente y se difunda de la manera más amplia posible

Con metadatos ajustados a la normativa internacional

Aplicaciones Linked Open Data (LOD) con Reconciliación Semántica

Aplicaciones que pueden incorporar Recolector y Repositorio OAI-PMH

Aplicaciones pensadas y desarrolladas para hacer más eficiente y ágil el trabajo de los administradores y más comprensible para el usuario el acceso a los recursos catalogados. 